\title{
Moving shadows on the dusty disks of young stars
}

\author{
L. V. Tambovtseva ${ }^{1,2}$, V. P. Grinin ${ }^{1,2,3}$, and G. Weigelt ${ }^{2}$
}

1 Main Astronomical Observatory Pulkovo, Pulkovskoe shosse 65, St. Petersburg 196140, Russia e-mail: tamb@gao.spb.ru; grinin@vg1723.spb.edu

2 Max-Planck-Institute für Radioastronomie, Auf dem Hügel 69, 53121 Bonn, Germany e-mail: weigelt@mpifr-bonn.mpg.de

3 Crimean Astrophysical Observatory, Crimea, Nauchny, Ukraine

Received 22 July 2005 / Accepted 25 October 2005

ABSTRACT

We investigate the formation of moving shadows on the circumbinary (CB) disk of young binary systems. Moving shadows can be created by a dusty disk wind of the secondary component. The densest parts of the dusty disk wind and the associated common envelope can be optically thick and may block the stellar radiation inside a certain solid angle, resulting in the appearance of a moving shadow zone. Its shape and size depends on the mass loss rate, the disk wind velocity, and optical properties of the dust. Our calculations show that the shadow zone is observable if the mass loss rate $\dot{M}_{\mathrm{w}}$ is greater than $10^{-9} M_{\odot}$ per year. This shadow resembles a clock hand. If the orbit is an elliptical, the properties of this clock hand will change during the orbital motion of the secondary.

Key words. accretion, accretion disks - stars: formation - binaries: close - circumstellar matter - stars: pre-main sequence

\section{Introduction}

During the last decade, high-resolution images of the dust environment of young stellar objects (YSOs) (e.g., Burrow et al. 1996; Koresko 1998; Padgett et al. 1999; Stapelfeldt et al. 1999) have stimulated numerous theoretical investigations. Most of the model images are calculated for the case of a single young star (e.g., Whitney \& Hartmann 1992, 1993; Wolf et al. 2003; Grosso et al. 2003; Hodapp et al. 2004). The observed images show different types of asymmetries. For example, Duchêne et al. (2004), Krist et al. (2002), and Itoh et al. (2002) report on images of the circumbinary disk of GG Tau (HBC 54; IRAS 04296+1725). The images show a ring-shaped CB disk with a small gap that could be a shadow caused by material between the stars and the ring. Roberge et al. (2004) present the first spatially resolved spectrum of scattered light from the TW Hydrae protoplanetary disk. The radial profile of the integrated disk brightness showed an azimuthal asymmetry that was not seen in the previous coronagraphic images.

In this paper, we investigate the formation of moving shadows on the CB disks of young binaries. Our analysis is based on the results of our previous papers (Grinin \& Tambovtseva 2002; GT2002; Grinin et al. 2004; GTS2004), in which we investigated the effect of a common envelope, created by a dusty disk wind of the secondary, on the optical variability of the system. It is shown that the densest part of the common envelope may be optically thick and may periodically obscure the main component. Furthermore, this dusty disk wind can cause a moving shadow on the surfaces of the CB disk. We calculated the CB disk images for several disk wind models, and the results are compared with observations of YSOs.

\section{Modeling}

The hydrodynamic (SPH) simulations reported by Artymowicz \& Lubow (1996) (AL96) show that in young binaries, the mass accretion rate from the $\mathrm{CB}$ disk onto the low-mass companion can exceed the mass accretion onto the main component. Bate \& Bonnell (1997) and Roẑycka \& Laphlin (1997) then confirmed this conclusion. According to them, the mass accretion rate ratio $q=\dot{M}_{2} / \dot{M}_{1}$ strongly depends on the specific angular momentum $j_{a}$ of the accreting matter and can exceed one order of magnitude in systems where the mass ratio $m_{2} / m_{1} \ll 1$, if $j_{a}$ is greater than the orbital specific angular momentum of the secondary.

The accretion activity in young binaries is a periodic function of time (AL96), and the accretion is maximum near the apoastron passage. The amplitude of modulation of the mass accretion rate onto components $\dot{M}_{i},(i=1,2)$ depends on eccentricity $e$ (AL96) and also on the viscosity (Roẑyczka \& Laughlin 1997). One might expect that the mass loss rates caused by the disk winds $\dot{M}_{\mathrm{w}}$ on the components will also vary periodically. However, this is not the case. Due to the large hydrodynamic time scale $t_{\text {hyd }}$, which is much greater than the orbital period, the orbital variations of $\dot{M}_{\mathrm{w}}$ will be very weak (GTS2004). Therefore, we only consider systems with constant mass loss rates. 


\subsection{The disk wind parameters}

The main source of information for the velocity field in disk winds of young stars are observations of forbidden lines ([OI] $6300 \AA$ and some others) in the spectra of young stars (Hartigan et al. 1995; Hirth et al. 1997) and the results of theoretical modeling (e.g., Goodson et al. 1999). According to Hartigan et al. (1995) and Hirth et al. (1997), the typical radial velocities of the low-velocity component of disk winds in the region of the forbidden line formation ( $\geq 1 \mathrm{AU}$ ) range from about $5 \mathrm{~km} \mathrm{~s}^{-1}$ to a few tens of kilometers per second. In a binary system with a star of one solar mass as the main component, these characteristic velocities are on the order of the Keplerian velocity of the secondary's orbital motion, for semimajor axes in the range of $\approx 1$ to $10 \mathrm{AU}$.

We consider the disk wind of the secondary to be an axially symmetric bipolar outflow in its own coordinate system ${ }^{1}$. The outflow is assumed to be isotropic within the wind ejection angle range from $\omega_{\min }$ to $\omega_{\max }$, where the wind ejection angle is the angle between the disk symmetry axis and the direction in which wind particles are ejected from the accretion disk of the secondary. For simplicity, we assume that the wind density and velocity do not depend on the latitude within the above angle range. We assume that the ejection angle range lies between about 40 to 60 degrees. In this range the main part of the low-velocity component of the disk wind is formed (Goodson et al. 1999). According to the numerical results of these authors, the low-velocity component provides the biggest contribution (about $80 \%$ ) to the mass loss rate of the disk wind. Therefore, the high-velocity component is not important in the models considered below.

Safier (1993) introduced a generalized version of the Blandford \& Payne (1982) self-similar wind model and argued that the disk-driven outflow had enough momentum to lift dust grains from the disk surface. He showed that the largest (millimeter-sized) dust grains are not lifted up by the outflow. Following Safier (1993) and Garcia et al. (2001), we assume that the disk wind contains both gas and dust components and that they have a "standard" proportion, as in the interstellar medium (100:1). We also assume that the dust component of the wind consists of a mixture of dust particles similar to those in the circumstellar (CS) disks of TTSs (Men'shchikov et al. 1999). According to this paper, the absorption coefficient of such a mixture is equal to $\approx 5 \times 10^{4} \mathrm{~cm}^{2} \mathrm{~g}^{-1}$ per unit of the dust mass at the wavelength of $5500 \AA$ ( $V$ band). In accordance, the absorption coefficient per unit of the gas and dust mixture is $\kappa_{V}=500 \mathrm{~cm}^{2} \mathrm{~g}^{-1}$.

\subsection{The common envelope}

We calculate the trajectories of the particles that are ejected by the low-mass companion during its orbital motion along its eccentric orbit in the gravitational field of the primary. In all models we considered the primary with the one solar mass and the semimajor axis equal to $3 \mathrm{AU}$. As in our previous papers (see

\footnotetext{
1 Assuming the axial symmetry of a secondary's disk wind means that we neglect the possible influence of the main component's tidal forces on the wind formation.
}

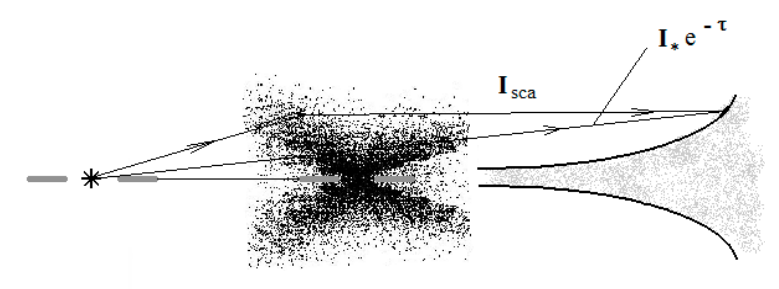

Fig. 1. Illustration of the geometry of the model. An asterisk indicates the location of the primary. The secondary is not seen because of the disk wind above and below the circumsecondary disk. The drawing is not to scale.

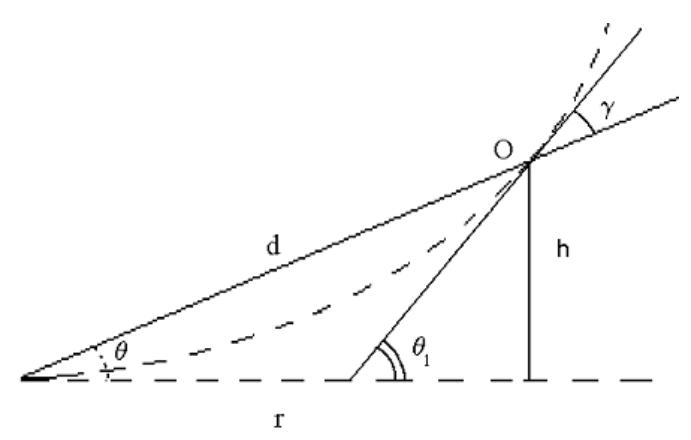

Fig. 2. Disk geometry (see text for details).

e.g. GT2002), we used the ballistic approximation for which the motion of each particle is independent of the others. The radial and azimuthal velocity components $V_{\mathrm{w}}$ and $U_{\mathrm{w}}$ are the input parameters of the modeling (GT2002). The distribution of the initially isotropic particles (in the secondary rest frame) becomes anisotropic because of the orbital motion of the windejecting secondary. The motion of each particle was calculated until it escaped the system or when the particle reached the equatorial plane of the binary. A steady-state mass distribution in the central part of the common envelope is obtained after about ten orbital periods. A sketch illustrating the geometry of the model is shown in Fig. 1.

\subsection{The shadows on the disks}

To model CB disk images, we use a simple model of a flared passive disk. This approximation cannot be used for the innermost regions of the $\mathrm{CB}$ disk, where the gravity perturbations caused by the orbital motion of the components are strong and produce density waves (Lin \& Papaloizou 1993; Lubow \& Artymowicz 2000). However, it is a good approximation for the outer regions of a $\mathrm{CB}$ disk where the binary system can be considered as a single star with a mass equal to the total mass of the system. Figure 2 shows a schematic picture of the flared disk model, in which the surface and midplane are shown as dashed lines. An arbitrary point $\mathrm{O}$ on the disk surface has a height $h$ and a projected radial distance $r$ in the midplane. The distance between the center of the star and a point $\mathrm{O}$ is $d^{2}=r^{2}+h^{2}$. There $\theta$ is the angle between the midplane and the 
line through $\mathrm{O}$ and the center, and $\theta_{1}$ is the angle between the midplane and the tangential line to the disk surface at point $\mathrm{O}$.

Figure 2 shows that

$$
\theta=\arctan \frac{h}{r} ; \quad \theta_{1}=\arctan \frac{\mathrm{d} h}{\mathrm{~d} r} ; \quad \gamma=\theta_{1}-\theta .
$$

We used a commonly accepted representation of the disk scale height $h(r)=h_{0}\left(\frac{r}{r_{0}}\right)^{\beta}$, where $h_{0}$ was assumed to be equal to $0.1 r_{0}$, the inner radius $r_{0}$ is assumed to be three-times the semimajor axis of the elliptic binary orbits, and $\beta$ is a free parameter. In our calculations, we used $\beta=9 / 8$ and 5/4 (Kenyon $\&$ Hartmann 1987; D’Alessio et al. 1999).

Equation (1) can be rewritten as:

$$
\frac{h}{r}=\frac{h_{0}}{r_{0}}\left(\frac{r}{r_{0}}\right)^{\beta-1} \text {. }
$$

Then we obtain

$$
\frac{\mathrm{d} h}{\mathrm{~d} r}=\beta \frac{h_{0}}{r_{0}}\left(\frac{r}{r_{0}}\right)^{\beta-1} .
$$

The radiation flux at the point $\mathrm{O}$ on the disk surface can be described as:

$F(r, \phi)=\frac{L_{*}}{4 \pi d^{2}} \sin \gamma$,

where $L_{*}$ is the luminosity of the star. Taking into account that part of the radiation flux will be occulted by the common envelope created by the disk wind of the secondary companion, we obtain

$F(r, \phi)=\frac{L_{*} \mathrm{e}^{-\tau(r, \phi)}}{4 \pi\left(r^{2}+h^{2}\right)} \sin \gamma$,

where $\phi$ is an azimuthal angle, and $\tau(r, \phi)$ is the optical thickness of the common envelope on the way between the main component and the point $r, \phi$ on the disk. A detailed description of the calculations of the common envelope's optical properties is given in GT2002.

The scattered radiation from the disk surface is assumed to be proportional to $F(r, \phi)$. This is a good approximation if the free-path length of photons in the disk is small compared to the size of the shadow. In the CS disks of the pre-main-sequence stars, this condition holds everywhere except at the periphery of the disks. We did not take the forward scattering of the stellar radiation by the disk wind into account since the albedo of dust particles is low, $\sim 0.4-0.5$ (Natta \& Whitney 2000). In order to demonstrate its possible effect on the contrast in the shadow, we calculated the scattered light for one of the wind models in the single scattering approximation.

Using this approach for accretion disks and the phasedependent density distribution obtained earlier for the common envelopes (GT2002; GTS2004), we calculated disk images seen face-on for several disk wind models.

\section{Results and discussion}

Table 1 presents the parameters of our models: eccentricity of the orbit, two velocity components of the wind (radial $V_{\mathrm{w}}$ and
Table 1. The model's parameters.

\begin{tabular}{lllll}
\hline \hline Models & $e$ & $V_{\mathrm{w}}$ & $U_{\mathrm{w}}$ & $\omega$ \\
\hline 1 & 0.5 & 1 & 0.5 & $40-60$ \\
2 & 0 & 3 & 0 & 45 \\
3 & 0.5 & 2 & 0 & $40-60$ \\
4 & 0.5 & 2 & 1 & $40-60$ \\
\hline
\end{tabular}

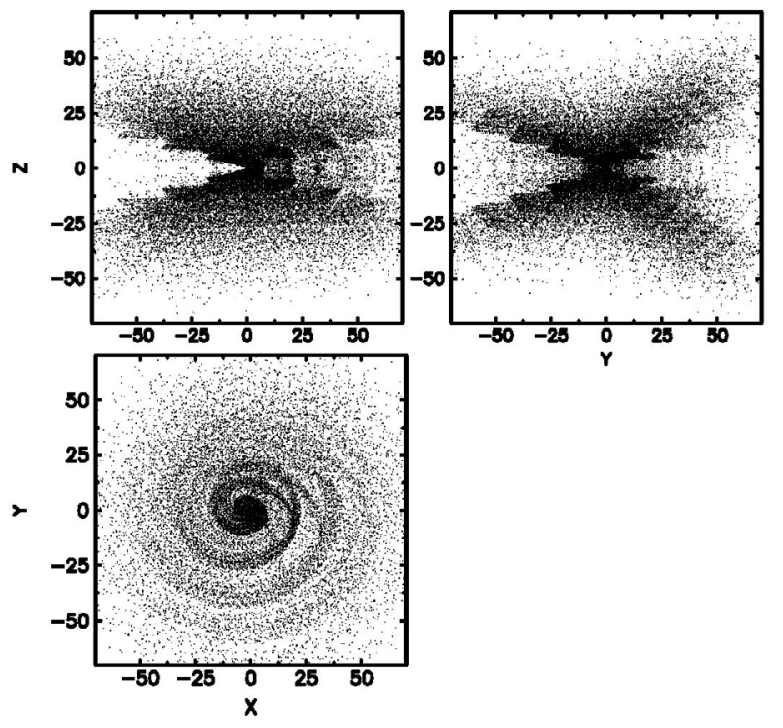

Fig. 3. Matter distribution in the common envelope in the $X Z$ and $X Y$ plane for model 1 . The semimajor axis of the binary coincides with the $X$-axis. The low-mass companion is at point with coordinates $X=0.5 ; Y=0$.

tangential $U_{\mathrm{w}}$ ) in units of the Keplerian velocity of the secondary in the periastron, and the wind ejection angle $\omega$ in degrees.

Figure 3 shows, as an example, the matter distribution in the common envelope for model 1. Deviations from the axial symmetry, which are caused by the vector summing of the wind and orbital velocities mentioned above, are clearly seen in the $X Z$ and $Y Z$ images, as well as the asymmetric spiral structure of the pole-on $(X Y)$ image. The secondary is at the point $X=0.5$ and $Y=0$. All distances are given in the units of the semimajor axis.

In Figs. 4-7, we present our calculated intensity distributions for models 2-4 in the assumption that the disk surface isotropically irradiates an incident light. All brightness contours are given in arbitrary units (the contours are spaced by a factor of 0.2). The coordinates $X$ and $Y$ are expressed in units of the orbital semimajor axis. Figure 4 shows the isophotes computed for model 2 . The disk flaring parameter $\beta=9 / 8$. We plotted three maps with different mass loss rates: $3 \times 10^{-8}$, $10^{-8}$, and $3 \times 10^{-9} M_{\odot}$ per year. One can see that a noticeable shadow appears on the disk surface if the mass loss rate is significant enough ( $>10^{-9} M_{\odot}$ per year). Figure 5 shows the poleon view of the disk with a shadow for two different parameters $\beta$. Evidently, more flared disks show wider shadows at the disk periphery. The centers of the images are masked with filled circles since the central regions of the common envelopes can 


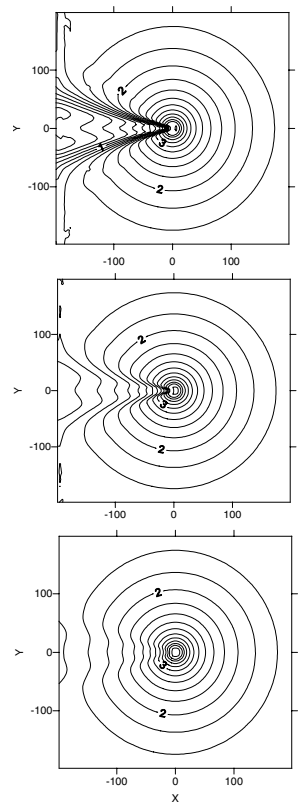

Fig. 4. Theoretical brightness map for model 2: $\beta=9 / 8$. From top to bottom: $\dot{M}_{\mathrm{w}}=3 \times 10^{-8}, 10^{-8}$, and $3 \times 10^{-9} M_{\odot}$ per year. The numbers on the curves mark the intensities in the logarithmic scale, and the contours are spaced by a factor of 0.2 .
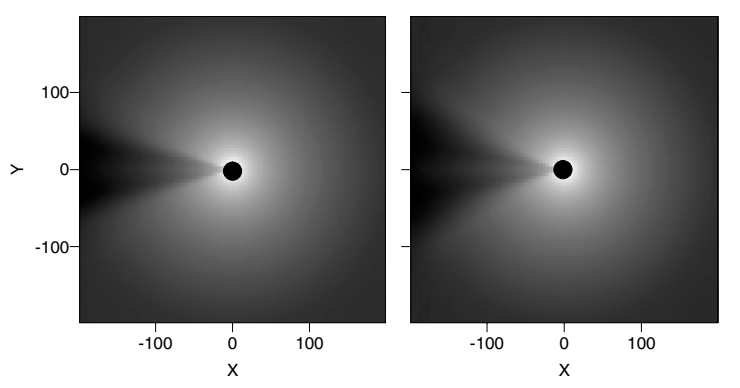

Fig. 5. Theoretical brightness maps for model $2: \beta=9 / 8$ (left), $\beta=$ $5 / 4($ right $), \dot{M}_{\mathrm{w}}=10^{-8} M_{\odot}$ per year.

cause additional scattering (GTS2004), which is not taken into consideration in the present paper; but this scattering does not influence the shadow formation.

A more interesting situation is obtained in binary systems with elliptic orbits (Figs. 6 and 7 corresponding to models 3 and 4). In this case, the shape of the shadow on the disk will change during the orbital motion: at the moment of the apoastron passage, the shadow will be narrower and fainter than in the periastron. In the periastron, the shadow can be much more extended (right columns of Figs. 6, 7); in Figs. 6 and 7, we show the disk with a cut outer region to represent its inner part better.

As an example, Fig. 8a illustrates the decrease in the shadow's intensity with the distance from the star calculated for model 3 . Both intensity profiles are given at the distances of 30 and $300 \mathrm{AU}$. The mass loss rate due to the wind is $10^{-8} M_{\odot} \mathrm{yr}^{-1}$. This case is shown when the secondary component is in the apoastron. Intensities are normalized to their maximum values calculated out of the shadow (in the bright regions). The shadow profile has a double-peaked form, which
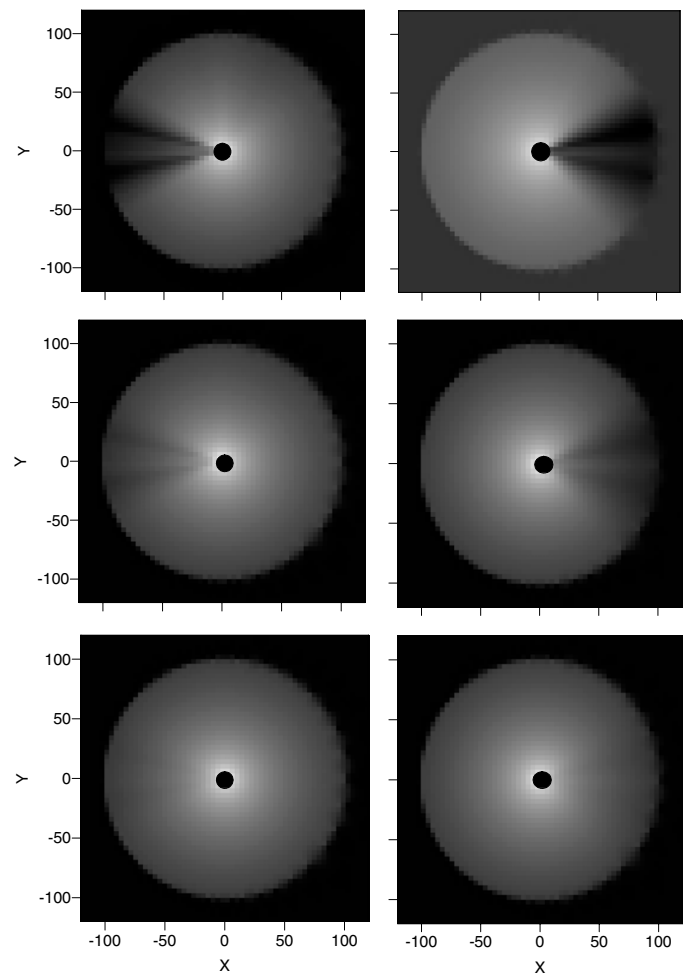

Fig. 6. Theoretical brightness maps for model 3: $\beta=5 / 4$, (left) apoastron, (right) periastron; from top to bottom: $\dot{M}_{\mathrm{w}}=3 \times 10^{-8}, 10^{-8}$ and $3 \times 10^{-9} M_{\odot}$ per year.

is also seen on the most images presented in Figs. 4-7. It is caused by a specific wind geometry: the particles are ejected within a cone limited by angles of 40 and 60 degrees.

Since the dust opacity depends on the wavelength, the contrast is also sensitive to the wavelength. Figure $8 \mathrm{~b}$ shows an azimuthal intensity profiles of the $\mathrm{CB}$ disk for the disk wind model 3 in the two bands: $V$ and $H$. The cut is made at the distance of $30 \mathrm{AU}$ from the star. Opacity in the $H$ band is less than in the $V$ band by about one order of magnitude. Therefore, a contrast between shadowed and bright parts of the CB disk dramatically decreases to the longer wavelengths. This means that in the near infrared the wind shadow can be detected for greater mass loss rates $\left(>10^{-8} M_{\odot} \mathrm{yr}^{-1}\right)$.

Figure 9 demonstrates changes in the shadow's shape and depth during the orbital period depending on the position of the secondary on the orbit. For a better representation we combine shadow profiles for the periastron and the apoastron positions. One can see that the "periastron shadow" is wider and deeper than the "apoastron" one, which is due to the fact that the particle concentration in the disk wind decreases with the distance from the source of the wind. For the same reason a contrast of the shadow from the disk wind will decrease with increase in the semimajor axis of the orbit. The results show that a shadow on the $\mathrm{CB}$ disk of a young binary system resembles a clock hand. In the case of an elliptical orbit, the properties of this clock hand, including its shape, can change during the orbital motion of a secondary (Fig. 10). If the masses (and luminosity) of the companions are similar, their disk winds can form two shadows that are located opposite each other. 

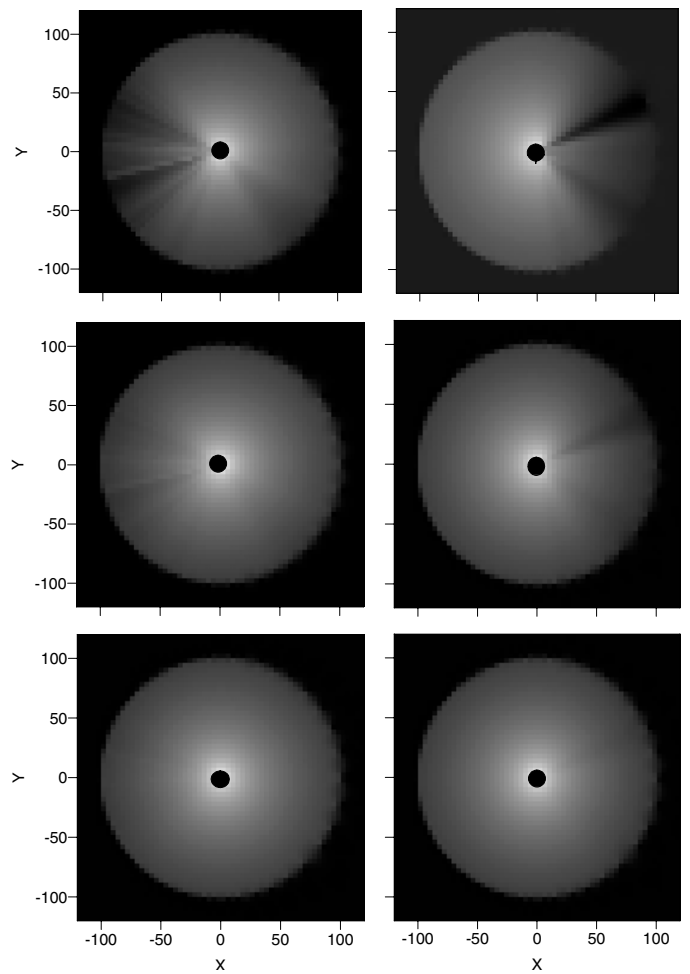

Fig. 7. Same as Fig. 6 but for model 4.
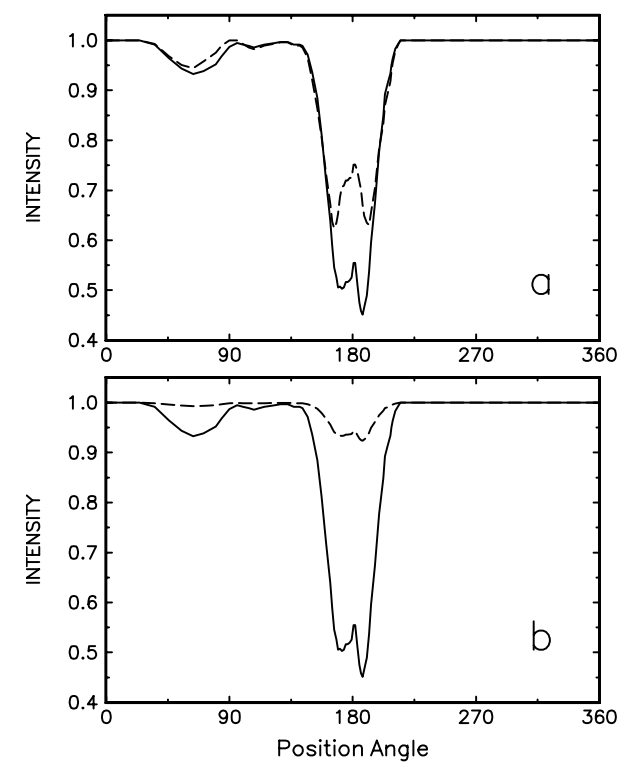

Fig. 8. a) Azimuthal profile intensity of the circumbinary disk in the $V$ band at distances of $30 \mathrm{AU}$ (solid) and $300 \mathrm{AU}$ (dashed) (model 3). b) $V$ band (solid) and $H$ band (dashed) azimuthal profile intensity of the circumbinary disk in the same model at 30 AU from the star. The mass loss rate $\dot{M}_{\mathrm{w}}=10^{-8} M_{\odot} \mathrm{yr}^{-1}$. All values are normalized to the corresponding maximum intensities taken far from a shadow.

For model 3 we calculated a radiation scattered by the disk wind for the optically semi-transparent case $\left(\dot{M}_{\mathrm{w}}=3 \times\right.$ $\left.10^{-9} M_{\odot} \mathrm{yr}^{-1}\right)$. In the single scattering approximation that is valid if the optical depth of the wind is small compared to unity ( $\tau \leq 0.5)$, the flux of the scattered radiation $F_{\text {sca }}$ from the whole

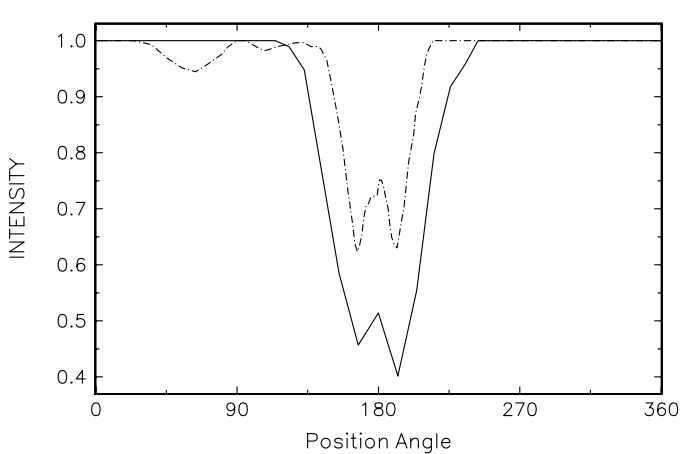

Fig. 9. Azimuthal variability of the $\mathrm{CB}$ disk intensity profile at $300 \mathrm{AU}$ from the star for the two positions of the secondary companion in model 3: a periastron (solid line) and an apoastron (dashed line). The mass loss rate $\dot{M}_{\mathrm{w}}=10^{-8} M_{\odot} \mathrm{yr}^{-1}$. All values are normalized to the corresponding maximum intensities taken far from a shadow.

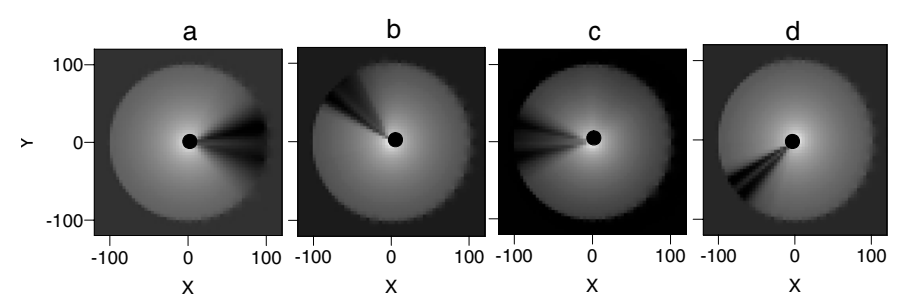

Fig. 10. Shadow motion. a) periastron, c) apoastron, b), d) phases between periastron and apoastron.

disk wind falling on the disk surface at a given point can be written as

$F_{\text {sca }}=\int_{V} \frac{L_{*}}{4 \pi d_{1}^{2}} \frac{1}{4 \pi d_{2}^{2}} \rho_{\mathrm{w}} \kappa_{\mathrm{sca}} e^{-\left(\tau_{1}+\tau_{2}\right)} f_{\mathrm{HG}}(\alpha) \cos \xi \mathrm{d} V$,

where $d_{1}$ is the distance from the star to the arbitrary point in the wind, $d_{2}$ the distance from this point to that on the disk, $\tau_{1}$ and $\tau_{2}$ are optical depths along $d_{1}$ and $d_{2}, \rho_{\mathrm{w}}$ is the mass density, $\kappa_{\mathrm{sca}}$ a scattering opacity in $\mathrm{cm}^{2}$ per gram, $f_{\mathrm{HG}}(\alpha)$ the Henyey-Greenstein phase function $\left(f_{\mathrm{HG}}(\alpha)=\left(1-g^{2}\right) /\left(1+g^{2}-\right.\right.$ $\left.2 g \cos \alpha)^{3 / 2}\right), g$ the asymmetry factor, $\alpha$ the scattering angle, and $\xi$ the angle between a direction of the scattered radiation from the arbitrary point in the wind to a given point on the CBdisk and a normal to the disk surface at this point. Integration is carried out over a whole wind volume $V$. Calculations were made for the $V$-band with the disk flaring parameter $\beta=5 / 4$, $g=0.5$; the albedo was taken to be equal to 0.5 to determine $\kappa_{\text {sca }}$. Figure 11 shows an azimuthal behavior of the $\mathrm{CB}$ disk intensity at the distances of $30 \mathrm{AU}$ (a) and $150 \mathrm{AU}$ (b) from the primary with and without the scattered radiation from the disk wind. This case is considered when the secondary is in the periastron. One can see that a shape and a depth of the shadow do not significantly depend on the radiation scattered by the disk wind; its contribution to the disk surface intensity at $30 \mathrm{AU}$ is about $5 \%$ in the bright region and $14 \%$ in the shadow zone. Analogous ratios at $150 \mathrm{AU}$ are $\sim 2 \%$ and $5 \%$, respectively. For the optically thicker winds, a major part of the stellar radiation will be absorbed and the fraction of the scattered radiation will be even less because of the low albedo of the dust. As seen from Fig. 11, an effect of scattering by the wind also leads to a small brightening of the disk surface around the shadow. 


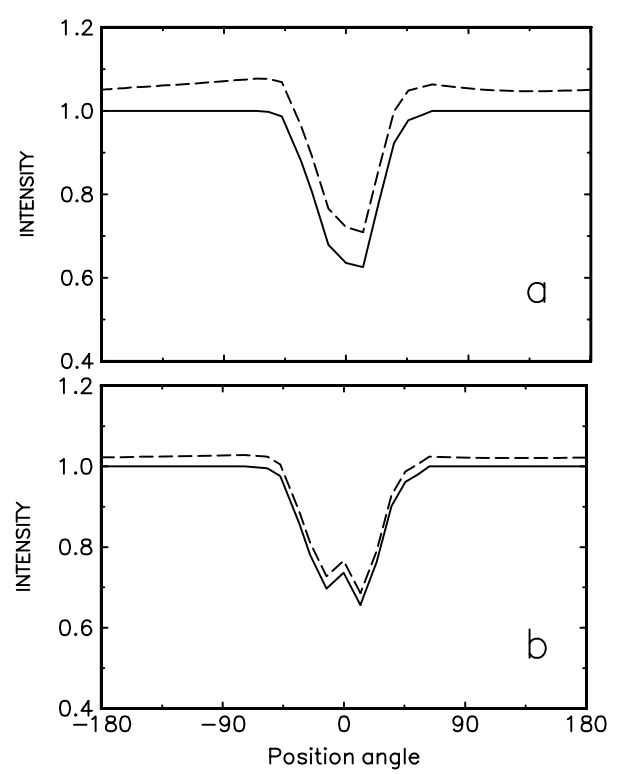

Fig. 11. Azimuthal profile of the CB disk intensity without (solid) and with (dashed) radiation scattered by the disk wind for model 3 at the distances of $30 \mathrm{AU}$ a) and $150 \mathrm{AU} \mathbf{b})$ from the star. The secondary is in the periastron. The mass loss rate $\dot{M}_{\mathrm{w}}=3 \times 10^{-9} M_{\odot} \mathrm{yr}^{-1}$. The curves are scaled to the corresponding maximum intensities of the CB disk without scattered radiation.

In some cases, $\mathrm{CS}$ disks around companions may produce their own shadows on the outer $\mathrm{CB}$ disk. But accretion disks are geometrically thin $(h / r \ll 1)$. Besides, they are rather compact (Artymowicz \& Lubow 1994). The noticeable shadows they cause may be seen only under specific conditions, such as when the primary is an AA Tau-like star. The inner part of the accretion disk around this star is warped due to interaction with the stellar magnetosphere formed by an inclined magnetic dipole (Bouvier et al. 2003; Terquem \& Papaloizou 2000). The shadows from such a warped disk will also be moving but with a period close to that of the star rotation.

\subsection{The shadows on images of YSOs}

The asymmetry of disks and outflows of several young objects were explained by shadow effects. One of them is the T Tau star GG Tau, which is a well-studied multiple system consisting of two binaries: GG Tau A and GG Tau B, while GG Tau A's $\mathrm{CB}$ disk was spatially resolved in the several wavelengths. The HST (Silber et al. 2000; Krist et al. 2002) and Subaru images (Itoh et al. 2002) show a narrow gap in GG Tau A's CB disk. The origin of this gap is a matter of debate. Krist et al. (2002) assume that the gap could be a shadow caused by a dust structure between the disc and the primary component. However, this shadow is not located on the line through the two binary components, but in a direction that is nearly perpendicular to this line. Therefore, we cannot conclude that this gap is caused by the disk wind of the secondary.

Another possibility is that the gap is the shadow of an opaque CS dust cloud. Such clouds exist in the vicinity of young stars and can be responsible for their brightness variability. This mechanism can explain the variability of the UX Ori type stars (e.g., Grinin 2000). However, if this mechanism were the cause of GG Tau A's disk gap, then the gap position should change during the orbital motion of the CS dust cloud. Since GG Tau A's disk gap did not change for several years, the origin of GG Tau A's disk gap is still a mystery.

$\mathrm{HH} 30$. One of the most spectacular asymmetric objects is the reflection nebula of the jet object HH 30 (Stapelfeldt et al. 1999). Asymmetry was present in 1998, but was absent in the 1994 and 1995 images. Wood \& Whitney (1998) consider the possibility that such asymmetry could be caused by a variable, asymmetric illumination of the CS disk by a rotating T Tauri star with spots on its surface. Cool spots could be the result of a large-scale magnetic field, and hot spots could be caused by accretion activity controlled by the stellar magnetic field. If the spots are asymmetrically distributed around the stellar axis, the rotation of the star leads to a variable illumination of the surrounding CS matter and can produce the changing asymmetric image shapes. Stapelfeld et al. (1999) consider this model for the interpretation of HH 30's variable asymmetry. Another possible explanation discussed by Stapelfeldt et al. (1999) is a variable CS extinction of the direct stellar radiation caused by opaque dust clouds. If HH 30 is a close binary, a dusty disk wind of the secondary could play the role of such an eclipsing cloud. Other young objects with very asymmetric inner diskoutflow environments are, for example, R Mon (e.g., Weigelt et al. 2002) and S 140 IRS 3 (Preibisch et al. 2001).

\section{Conclusion}

The main properties of the model considered in this paper can be summarized as follows:

- The densest parts of the dusty disk wind and associated common envelope can be optically thick and may block the stellar radiation inside a certain solid angle. This results in the appearance of a moving shadow zone whose shape and size depend on the mass loss rate, disk wind parameters, and the optical properties of the dust. The shadow zone is observable if the mass loss rate is higher than $10^{-9} M_{\odot}$ per year in the visible and higher than $10^{-8} M_{\odot}$ per year in the near-infrared. The shadow of the disk wind is a permanent feature of a CB disk. In the case of an elliptic orbit, the shadow on the $\mathrm{CB}$ disk changes its shape during the orbital motion of the secondary. The shadow is less extended and fainter during the apoastron phase.

- Detection of moving shadows on the disks of young stars can provide new information for studies of young binary systems. Such observations might also be very interesting for studying the disk winds themselves. The pole-on orientation of the disks is most suitable for such observations, but shadows can also be observed on disks seen edge-on. Optical imaging monitoring on long and short timescales would be very interesting when seeking moving shadows. If a shadow is caused by the disk wind of a secondary, it will change its position on the time scale equal to the orbital period of a system (in years).

Acknowledgements. V.G. and L.T. acknowledge the kind hospitality of the Max-Planck-Institute für Radioastronomie, where part of this 
work has been done. We are grateful to the anonymous referee for useful comments and suggestions. This project was supported in part by the grant of the Russian Academy of Sciences "Non-stationary phenomena in Astronomy" and the grant INTAS 03-51-6311.

\section{References}

Artymowicz, P., \& Lubow, S. H. 1994, ApJ, 421, 651

Artymowicz, P., \& Lubow, S. H. 1996, ApJ, 467, L77

Bate, M. R., \& Bonnel, I. A. 1997, MNRAS, 285, 33

Blandford, R. D., \& Payne, D. G. 1982, MNRAS, 199, 883

Bouvier, J., Grankin, K. N., Alencar, S. H. P., et al. 2003, A\&A, 409, 169

Burrows, C. J., Stapelfeldt, K. R., Watson, A. M., et al. 1996, ApJ, 473, 437

D’Alessio, P., Calvet, N., Hartmann, L., Lizzons, S., \& Cantó, J. 1999, ApJ, 527, 893

Duchêne, G., McCabe, C., Ghez, A. M., \& Macintosh, B. A. 2004, ApJ, 606, 969

Garcia, P. J. V., Ferreira, J., Cabri, S., \& Binette, L. 2001, A\&A, 377, 589

Goodson, A. P., Böhm, K. H., \& Winglee, R. 1999, ApJ, 524, 142

Grinin, V. P. 2000, in Disks Planetesimals, and Planets, ed. F. Garz'on, C. Eiroa, D. de Winter, \& T. J. Mahoney (San Francisco: ASP), ASP Conf. Ser., 219, 216

Grinin, V. P., \& Tambovtseva, L. V. 2002, Astron. Lett., 28, 601

Grinin, V. P., Tambovtseva, L. V., \& Sotnikova, N. Ya. 2004, Astron. Lett., 30, 694

Grosso, N., Alves, J., Wood, K., et al. 2003, ApJ, 586, 296

Hartigan, P., Edwards, S., \& Ghandour, L. 1995, ApJ, 452, 736

Hirth, G. A., Mundt, R., \& Solf, J. 1997, A\&AS, 126, 437
Hodapp, K. W., Walker, C. H., Reipurth, B., et al. 2004, ApJ, 601, L79 Itoh, Y., Tamura, M., Hayashi, S. S., et al. 2002, PASJ, 54, 963

Kenyon, S. J., \& Hartmann, L. 1987, ApJ, 323, 714

Koresko, C. D. 1998, ApJ, 507, L145

Krist, J. E., Stapelfeldt, K. R., \& Watson, A. M. 2002, ApJ, 570, 785

Lin, D. N. C., \& Papaloizou, J. C. B. 1993, in Protostars and Planets III, ed. E. H. Levy, \& J. I. Lunine (Tucson: Univ. Arizona Press), 749

Lubow, S. H., \& Artymowicz, P. 2000, in Protostars and Planets IV, ed. V. Mannings, A. P. Boss, \& S. S. Russel (Tucson: Univ. Arizona Press), 731

Men'shchikov, A. B., Henning, T., \& Fisher, O. 1999, ApJ, 519, 257

Natta, A., \& Whitney, B. A. 2000, A\&A, 364, 633

Padgett, D., Brandner, W., Stapelfeldt, K. R., et al. 1999, AJ, 117, 1490

Preibisch, T., Balega, Yu. Yu., Schertl, D., Smith, M. D., \& Weigelt, G. 2001, A\&A, 378, 539

Roberge, A., Weinberger, A. J., \& Malumuth, E. M. 2004 [arXiv: astro-ph/0410251]

Róẑyczka, M., \& Laughlin, G. 1997, in ASP Conf. Ser., 121, 792

Safier, P. N. 1993, ApJ, 408, 115

Silber, J., Gledhill, T., Duchêne, G., \& Ménard, F. 2000, ApJ, 536, L89

Stapelfeld, K. R., Watson, A. M., Krist, J. E., et al. 1999, ApJ, 516, L95

Terquem, C., \& Papaloizou, J. C. B. 2000, A\&A, 360, 1031

Weigelt, G., Balega, Yu. Yu., Hofmann, K.-H., \& Preibisch, T. 2002, A\&A, 392, 937

Whitney, B. A., \& Hartmann, L. 1992, ApJ, 395, 529

Whitney, B. A., \& Hartmann, L. 1993, ApJ, 402, 605

Wolf, S., Padgett, D. L., \& Stapelfeld, K. R. 2003, ApJ, 588, 373

Wood, K., \& Whitney, B. 1998, ApJ, 506, L43 